\title{
Antineutrino Charged-Current Reactions on Hydrocarbon with Low Momentum Transfer
}

R. Gran, ${ }^{1}$ M. Betancourt, ${ }^{2}$ M. Elkins, ${ }^{1,{ }^{*}}$ P. A. Rodrigues,${ }^{3,45}$ F. Akbar, ${ }^{6}$ L. Aliaga, ${ }^{7,8}$ D. A. Andrade, ${ }^{9}$ A. Bashyal, ${ }^{10}$ L. Bellantoni, ${ }^{2}$ A. Bercellie, ${ }^{5}$ A. Bodek,${ }^{5}$ A. Bravar, ${ }^{11}$ H. Budd,${ }^{5}$ G. F. R. Caceres Vera, ${ }^{12}$ T. Cai, ${ }^{5}$ M. F. Carneiro, ${ }^{10}$ D. Coplowe, ${ }^{3}$ H. da Motta, ${ }^{12}$ S. A. Dytman, ${ }^{13}$ G. A. Díaz,${ }^{5,8}$ J. Felix, ${ }^{9}$ L. Fields, ${ }^{2,14}$ R. Fine,${ }^{5}$ H. Gallagher, ${ }^{15}$ A. Ghosh, ${ }^{16,12}$ H. Haider, ${ }^{6}$ J. Y. Han, ${ }^{13}$ D. A. Harris, ${ }^{2}$ S. Henry, ${ }^{5}$ D. Jena,${ }^{2}$ J. Kleykamp, ${ }^{5}$ M. Kordosky, ${ }^{7}$ T. Le, ${ }^{15,17}$ J. R. Leistico, ${ }^{1}$ A. Lovlein, ${ }^{1}$ X.-G. Lu, ${ }^{3}$ E. Maher, ${ }^{18}$ S. Manly, ${ }^{5}$ W. A. Mann, ${ }^{15}$ C. M. Marshall, ${ }^{5, \dagger}$ K. S. McFarland, ${ }^{5,2}$ A. M. McGowan, ${ }^{5}$ B. Messerly, ${ }^{13}$ J. Miller, ${ }^{16}$ A. Mislivec ${ }^{5}$ J. G. Morfín, ${ }^{2}$ J. Mousseau, ${ }^{19, \$}$ D. Naples, ${ }^{13}$ J. K. Nelson, ${ }^{7}$ C. Nguyen, ${ }^{19}$ A. Norrick, ${ }^{7}$ Nuruzzaman, ${ }^{17,16}$ A. Olivier, ${ }^{5}$ V. Paolone, ${ }^{13}$ C. E. Patrick, ${ }^{14,8}$ G. N. Perdue,${ }^{2,5}$ M. A. Ramírez, ${ }^{9}$ R. D. Ransome,${ }^{17}$ H. Ray,${ }^{19}$ L. Ren, ${ }^{13}$ D. Rimal, ${ }^{19}$ D. Ruterbories, ${ }^{5}$ H. Schellman, ${ }^{10,14}$ C. J. Solano Salinas, ${ }^{20}$ H. Su, ${ }^{13}$ M. Sultana,${ }^{5}$ S. Sánchez Falero, ${ }^{8}$ E. Valencia, ${ }^{7,9}$ J. Wolcott, ${ }^{5, \|}$ M. Wospakrik, ${ }^{19}$ and B. Yaeggy ${ }^{16}$

(MINERvA Collaboration)

${ }^{1}$ Department of Physics and Astronomy, University of Minnesota-Duluth, Duluth, Minnesota 55812, USA

${ }^{2}$ Fermi National Accelerator Laboratory, Batavia, Illinois 60510, USA

${ }^{3}$ Oxford University, Department of Physics, Oxford, United Kingdom

${ }^{4}$ University of Mississippi, Oxford, Mississippi 38677, USA

${ }^{5}$ University of Rochester, Rochester, New York 14627 USA

${ }^{6}$ AMU Campus, Aligarh, Uttar Pradesh 202001, India

${ }^{7}$ Department of Physics, College of William \& Mary, Williamsburg, Virginia 23187, USA

${ }^{8}$ Sección Física, Departamento de Ciencias, Pontificia Universidad Católica del Perú, Apartado 1761, Lima, Perú

${ }^{9}$ Campus León y Campus Guanajuato, Universidad de Guanajuato, Lascurain de Retana No. 5, Colonia Centro, Guanajuato 36000, México

${ }^{10}$ Department of Physics, Oregon State University, Corvallis, Oregon 97331, USA

${ }^{11}$ University of Geneva, 1211 Geneva 4, Switzerland

${ }^{12}$ Centro Brasileiro de Pesquisas Físicas, Rua Dr. Xavier Sigaud 150, Urca, Rio de Janeiro, Rio de Janeiro 22290-180, Brazil

${ }^{13}$ Department of Physics and Astronomy, University of Pittsburgh, Pittsburgh, Pennsylvania 15260, USA

${ }^{14}$ Northwestern University, Evanston, Illinois 60208, USA

${ }^{15}$ Physics Department, Tufts University, Medford, Massachusetts 02155, USA

${ }^{16}$ Departamento de Física, Universidad Técnica Federico Santa María, Avenida España 1680 Casilla 110-V, Valparaíso, Chile

${ }^{17}$ Rutgers, The State University of New Jersey, Piscataway, New Jersey 08854, USA

${ }^{18}$ Massachusetts College of Liberal Arts, 375 Church Street, North Adams, Massachusetts 01247, USA

${ }^{19}$ University of Florida, Department of Physics, Gainesville, Florida 32611, USA

${ }^{20}$ Universidad Nacional de Ingeniería, Apartado 31139, Lima, Perú

(Received 23 March 2018; revised manuscript received 17 April 2018; published 1 June 2018; corrected 14 November 2018)

We report on multinucleon effects in low momentum transfer $(<0.8 \mathrm{GeV} / c)$ antineutrino interactions on plastic $(\mathrm{CH})$ scintillator. These data are from the 2010-2011 antineutrino phase of the MINERvA experiment at Fermilab. The hadronic energy spectrum of this inclusive sample is well described when a screening effect at a low energy transfer and a two-nucleon knockout process are added to a relativistic Fermi gas model of quasielastic, $\Delta$ resonance, and higher resonance processes. In this analysis, model elements introduced to describe previously published neutrino results have quantitatively similar benefits for this antineutrino sample. We present the results as a double-differential cross section to accelerate the investigation of alternate models for antineutrino scattering off nuclei.

DOI: 10.1103/PhysRevLett.120.221805

Published by the American Physical Society under the terms of the Creative Commons Attribution 4.0 International license. Further distribution of this work must maintain attribution to the author(s) and the published article's title, journal citation, and DOI. Funded by $\operatorname{SCOAP}$. 
Current and future accelerator-based neutrino oscillation experiments analyze flavor oscillations based on distortions of reconstructed antineutrino energy spectra. These measurements require models for both the lepton energy and angle, and for the hadronic system. Experiments using calorimetric reconstruction $[1,2]$ are especially sensitive to the presence of neutrons in the final state. To probe for charge-parity $(C P)$ violation in the lepton sector [3-5], models of antineutrino processes require similar accuracy to the corresponding neutrino processes. Otherwise, model uncertainties limit the sensitivity to, or possibly mimic, a $C P$-violating effect.

We present the first antineutrino analysis of inclusive charged-current reactions to isolate multinucleon effects in the quasielastic (CCQE) and $\Delta$ resonance kinematic regions. We reconstruct the hadronic system using calorimetry and obtain an estimate of the three-momentum transfer for each event. The data are subdivided into six subranges of momentum transfer up to $0.8 \mathrm{GeV} / c$, and within each range, we present the observed hadronic energy in the detector. To describe these data, a component of the event rate could be attributed to many-body effects like a two-particle, two-hole $(2 p 2 h)$ process [6-16]. Also, suppression of CCQE interactions is preferred, such as provided by a random phase approximation (RPA) calculation [7,17-19] applied to a Fermi gas model [20].

The data were taken with the NuMI beam [21] operating in antineutrino mode. The primary beam of $120 \mathrm{GeV}$ protons interacts in a graphite target, producing mesons. A pair of magnetic horns focuses negatively-charged mesons toward a decay pipe where their decay leads to an antineutrino spectrum in the MINERvA detector peaking near $3.0 \mathrm{GeV}$. We use a GEANT4-based [22,23] prediction for the flux with central values and uncertainties adjusted [24] using thin-target hadron production data [25-28] and an in situ neutrino-electron scattering constraint [29].

A sample of charged-current $\bar{\nu}_{\mu}$ interactions are selected from MINERvA's 5.3 ton fiducial volume by requiring that a muon track leaves the MINERvA detector and has its positive charge and momentum identified in the MINOS magnetized iron spectrometer [30] located $2 \mathrm{~m}$ downstream. The fiducial volume is both an active tracker and a calorimeter, built from planes of scintillator $(\mathrm{CH})$ strips with a triangular shaped $3.3 \mathrm{~cm}$ base and $1.7 \mathrm{~cm}$ height. Alternating and nesting the triangles gives light-sharing information that improves tracking resolution. Each hexagonal plane contains 127 strips of up to $245 \mathrm{~cm}$ in length. The planes are installed with strips oriented vertically or rotated $\pm 60^{\circ}$, ensuring the precise reconstruction of the interaction point and muon track angle, even when hadronic activity partially obscures the muon. The target mass consists of $8.2 \%, 88.5 \%$, and $2.5 \%$ hydrogen, carbon, and oxygen, respectively, plus small amounts of heavier nuclei.
Particles leaving the active tracking region pass into the electromagnetic calorimeter (ECAL), where thin sheets of lead are epoxied to each scintillator plane. Farther downstream are layers of hadronic calorimetry using alternating planes of scintillator and passive steel. The calorimetric and tracking capabilities of MINERvA are constrained relative to GEANT4 v.9.4.p2 (with the Bertini Cascade option) using in situ [31] and hadron test beam measurements [32]. With no test beam measurements, the neutron response and its uncertainties come after adjusting the cross section to match the data from [33] as used by later versions of GEANT4.

The kinematics of each event are reconstructed using the measured muon energy and angle, and measured energy deposits attributed to hadrons. The technique is nearly identical to [6]. A full simulation of the reconstructed sample with a calibrated detector response is made using the GEANT4 simulation and GENIE version 2.8.4 neutrino event generator [34]. This simulation is used to obtain a correction [35], as a function of the calorimetrically measured hadronic energy, to estimate the energy transfer $q_{0}$. This correction is applied identically to the reconstructed simulation and data. In both cases, the calorimetric neutrino energy estimate is $E_{\nu}=E_{\mu}+q_{0}$, where $E_{\mu}$ includes the muon rest mass $M_{\mu}$. The square of the four-momentum transfer is $-q^{2}=Q^{2}=2 E_{\nu}\left(E_{\mu}-p_{\mu} \cos \theta_{\mu}\right)-M_{\mu}^{2}$, and the three-momentum transfer is simply $q_{3}=\sqrt{Q^{2}+q_{0}^{2}}$. In this Letter, the kinematics of the analysis sample are limited to $q_{3}<0.8 \mathrm{GeV} / c$. There are no other requirements on reconstructed hadronic topologies for this inclusive sample.

The measured energy deposits are used to form another calorimetric estimator, the available energy $E_{\text {avail }}$ [6]. This is energy due to particles that deposit most or all of their energy in the detector: proton kinetic energy, charged pion kinetic energy, electrons, positrons, and photons, including those from neutral pion and eta decays. These momentum transfers are too low for the production of heavier mesons and baryons.

When $E_{\text {avail }}$ is formed from a model, it does not include neutrons that leave a small fraction of their energy in the detector or the energy used to unbind nucleons. In the neutrino case [6], where outgoing protons far outnumber neutrons, this is a good approximation. In the simulation of this antineutrino subsample, $70 \%$ of interactions have more than half of the energy transfer going to neutrons, including $40 \%$ which have neutron-only final states. Up to $60 \%$ of neutrons at these energies leave reconstructed energy deposits in the detector, so neutrons can contribute significantly to the hadronic energy deposits. Despite this, reconstructed or model distributions of $E_{\text {avail }}$ vs. $q_{3}$ retain the ability to separate CCQE and $\Delta$ resonance kinematics and the region between them. Because the analysis is limited to interactions with little energy in the recoil system, only the energy deposits in the tracker and downstream ECAL regions are considered. The backgrounds 


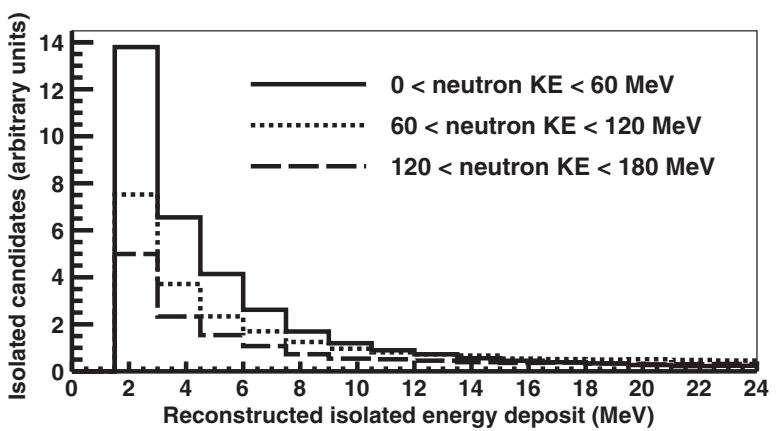

FIG. 1. Predicted isolated energy deposits from neutrons in three ranges of neutron kinetic energy (KE). These are selected from the tuned MC sample (described later) with $q_{3}<$ $0.8 \mathrm{GeV} / c$. The three curves also illustrate the relative abundance of lower kinetic energies in the selected MC sample. Neutrons in this energy range typically leave small isolated energy deposits, uncorrelated with the neutron kinetic energy.

from unrelated beam activity are higher and the calorimetric resolution is worse for energy deposits in the other regions, degrading the sensitivity to multinucleon effects.

While $E_{\text {avail }}$ is defined assuming neutrons have a negligible calorimetric response, the actual situation is more complex. Interactions that have only neutrons in the final state are most likely to have reconstructed hadronic energy between 0 and $10 \mathrm{MeV}$. Figure 1 shows the reconstructed energy deposits from the GENIE-produced neutrons exiting the nucleus and simulated by GEANT4 with the detector model. The most common outcomes are small $(<10 \mathrm{MeV})$ energy deposits as the neutrons scatter on hydrogen and carbon in the detector. The response is mostly uncorrelated with the neutron energy, and it is not suited for a calorimetric quantity. Larger secondary proton energy deposits become more common as neutron kinetic energy increases. For this analysis and its kinematic range, neutrons with tens to a few hundreds of $\mathrm{MeV}$ are effectively treated as biasing reconstructed $E_{\text {avail }}$ to higher values than the true quantity.

Another consequence of the neutron response in the MINERvA detector is that the resolutions for some reconstructed quantities are different than the neutrino case. The two hadronic energy estimators for the selected sample have significantly worse resolutions. The simulation indicates a root-mean-square (rms) resolution of 58\% for $q_{0}$, compared to $51 \%$ for the neutrino case in [6]. For $E_{\text {avail }}$, the rms is also $58 \%$, while the neutrino resolution is significantly improved to $40 \%$. When neutrons may be the only final state particle, the absolute residual is a better metric (shown in Fig. 2) than the fractional rms. The neutrino energy estimator is negligibly different; because these events had such little hadronic energy to begin with, the muon energy dominates the resolution. Muon energy and angle drive $q_{3}$. Its resolution is barely degraded from $22 \%$ to $23 \% \mathrm{rms}$, and it varies little across the range of $q_{3}$.

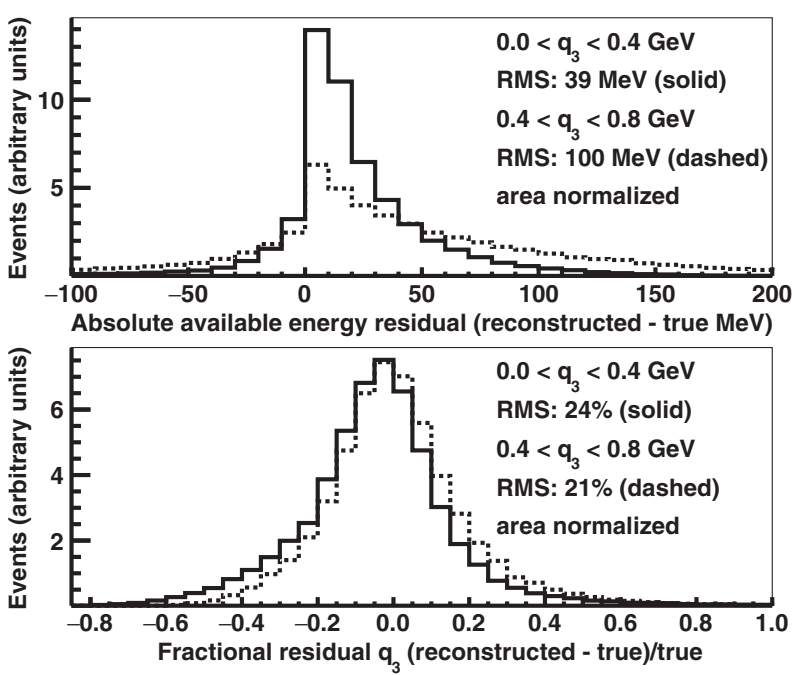

FIG. 2. Absolute resolution of $E_{\text {avail }}$ (top) and fractional resolution for three-momentum transfer (bottom), for the $\mathrm{MC}$ sample with reconstructed $q_{3}<0.8 \mathrm{GeV} / c$. The dashed line is the upper half of the $q_{3}$ range; the solid line is the lower half.

Because this is an analysis of an inclusive sample, event selection is minimal. We only create a boundary for unfolding the data into a double differential cross section that can be reproduced by external event generators, and we exclude regions of kinematic space that do not have good acceptance. The muon momentum is required to be above $1.5 \mathrm{GeV} / c$ and an angle less than 20 degrees with respect to the beam direction. We further limit the reconstructed antineutrino energy to between 2 and $6 \mathrm{GeV}$, which spans the peak of this beam and allows a direct comparison to the neutrino results [6]. These selections are used for the reconstructed events, the unfolded distribution, and the true distribution of MC simulations compared to the latter.

The selected inclusive sample is compared to the prediction of the GENIE event generator combined with a GEANT4 simulation of the outgoing particles from the reaction. GENIE's simulation of the CCQE process is from Llewellyn Smith [36], with vector form factors parametrized by [37], and the axial form factor taken to be a dipole with an axial mass of $0.99 \mathrm{GeV}$. For interactions on carbon and other nuclei, GENIE uses a Fermi gas model [20]. The $\Delta$ and higher resonances use Rein and Sehgal [38], with a nonresonant component taken from the deeply inelastic scattering model [39] as the resonances are phased out from invariant mass $1.4<\mathrm{W}<2.0 \mathrm{GeV}$. We add two minor (for this analysis $<2 \%$ of the total rate) modifications to pion production. The nonresonance, single-pion process is reduced to $43 \%$ of the nominal following the comparison of GENIE to bubble chamber experiment neutrino data $[40,41]$. Coherent pion events with pion kinetic energy $<0.45 \mathrm{GeV}$ are reduced by half [42-44]. This base combination of models, compared to reconstructed data, has discrepancies in the region between the $\mathrm{CCQE}$ and $\Delta$ process as large as a factor of two, as shown in the top panels of Fig. 3. 

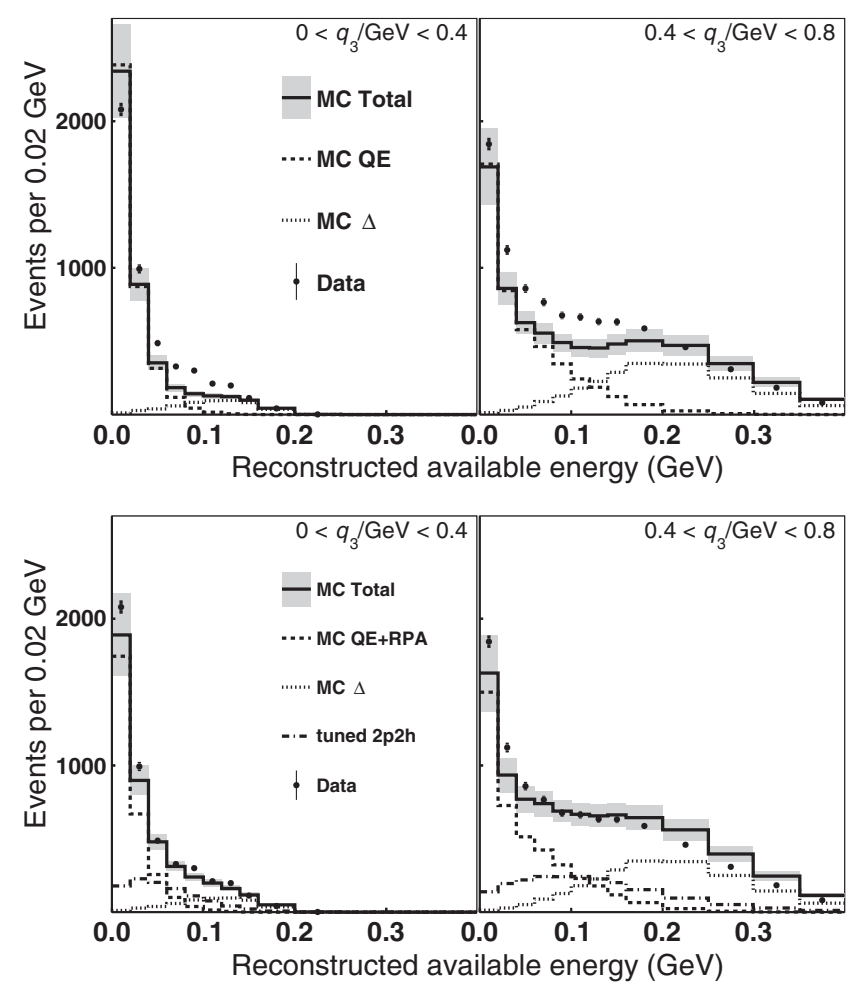

FIG. 3. Reconstructed $E_{\text {avail }}$ distributions compared to (top) the base Monte Carlo simulation (GENIE with minor modifications to pion production) for two ranges of reconstructed three momentum transfer. In the improved simulation MnvGENIE-v1 (bottom), the region between the predicted CCQE process (dashed) and the $\Delta(1232)$ resonance (dotted) is filled by events generated from the Valencia $2 p 2 h$ process plus additional $2 p 2 h$ events (dotdashed).

We have modified the default GENIE version 2.8.4 to include advances in modeling the important processes. The CCQE process is modified to include RPA screening based on the IFIC Valencia model $[17,45]$, implemented by weighting GENIE CCQE events [46]. A CCQE-like twoparticle, two-hole process " $2 p 2 h$," from the model by the same group [8,45], is implemented in GENIE [15].

The IFIC Valencia $2 p 2 h$ model increases the predicted event rates, but not enough. This process is increased further with an empirical enhancement [47] based on MINERvA inclusive neutrino data [6]. The additional events are from weighting up the generated $2 p 2 h$ events according to a two-dimensional Gaussian in true $q_{0}, q_{3}$, whose six parameters are fit to the neutrino data version of these distributions. This enhancement adds $50 \%$ to the predicted $2 p 2 h$ strength, but it targets the event rate in the kinematic region between the CCQE and $\Delta$ peaks where the rate doubles. The collection of changes in this and the preceding paragraphs are referred to as "MnvGENIE-v1" and are the central, tuned model for many recent analyses [48-51].

The resulting description of the antineutrino data is much improved, as illustrated in Fig. 3 and summarized in Table I
TABLE I. Comparison of the models to reconstructed data showing the evolution of the $\chi^{2}$ with each model change. The reconstructed data and the base model are as in the top panels, and the "tune" model is as in the lower panels of Fig. 3. The calculation actually uses the resolution-driven six bins of $q_{3}$ $\{0.0,0.2,0.3,0.4,0.5,0.6,0.8\} \mathrm{GeV} / c$ for best sensitivity, and they are summed into the same two ranges shown in Fig. 3. The right-most columns are made using the neutrino data [6] though the models being tested in this Letter have advanced since that earlier publication.

\begin{tabular}{|c|c|c|c|c|}
\hline $\begin{array}{l}\text { sample } \\
q_{3} \text { range } \\
\text { degrees of freedom }\end{array}$ & $\begin{array}{c}\bar{\nu}_{\mu} \\
\text { Lower } \\
19\end{array}$ & $\begin{array}{c}\bar{\nu}_{\mu} \\
\text { Upper } \\
37\end{array}$ & $\begin{array}{c}\nu_{\mu} \\
\text { Lower } \\
24\end{array}$ & $\begin{array}{c}\nu_{\mu} \\
\text { Upper } \\
41\end{array}$ \\
\hline $\begin{array}{l}\text { GENIE } 2.8 .4+\text { pion }[34,40-43] \\
+ \text { QERPA }[46,52] \\
+2 p 2 h[8,45] \\
+ \text { tune }[6,47]\end{array}$ & $\begin{array}{r}239 \\
261 \\
105 \\
69\end{array}$ & $\begin{array}{r}167 \\
140 \\
108 \\
80\end{array}$ & $\begin{array}{r}437 \\
265 \\
149 \\
77\end{array}$ & $\begin{array}{l}281 \\
253 \\
294 \\
150\end{array}$ \\
\hline $\begin{array}{l}\text { tune only } p n \text { initial state } \\
\text { tune not } p n \text { initial state } \\
\text { tune CCQE reactions }\end{array}$ & $\begin{array}{l}65 \\
71 \\
59\end{array}$ & $\begin{array}{r}86 \\
74 \\
123\end{array}$ & $\begin{array}{r}76 \\
84 \\
108\end{array}$ & $\begin{array}{l}160 \\
163 \\
166\end{array}$ \\
\hline +MINOS resonance tune [53] & 151 & 45 & 114 & 141 \\
\hline
\end{tabular}

using a standard $\chi^{2}$ test on the reconstructed samples. These models also improve the description of muon-only kinematic distributions of an overlapping subset of the same data set [50], selected with no pions in the final state.

For this model comparison to reconstructed data, the largest systematic uncertainties include flux, hadron energy scale, and GENIE resonance interaction and final-state rescattering model uncertainties. The GENIE uncertainty on the CCQE axial form factor is reduced to $\pm 9 \%$ following the analysis of [54]. An uncertainty on the RPA CCQE suppression [46,52] is added, most significantly from comparison to muon capture data. No single uncertainty dominates the model prediction for the reconstructed distributions.

The antineutrino sample retains a discrepancy just beyond the error band in the four second-lowest $E_{\text {avail }}$ bins within the range $0.3<q_{3}<0.8 \mathrm{GeV} / c$. These bins are dominated by events with neutron-only final states, including feed-down from higher energy transfer CCQE and $2 p 2 h$ reactions. Limited to the models available for this analysis, both the CCQE RPA and the tuned $2 p 2 h$ component each have a $10 \%$ to $30 \%$ effect on these bins. The comparison of the first two rows of Table I is subtle; the first does not contain additional uncertainty from the RPA model. Applying an estimate for the uncertainty to both rows also yields a worse $\chi^{2}$ for the lower $q_{3}$ range for neutrino when RPA is added. RPA reduces some bins where the MC simulation is already under predicting the data. However, the RPA model produces a better agreement in the lowest $E_{\text {avail }}$ for $0.0<q_{3}<0.3 \mathrm{GeV} / c$, which is also where the predicted RPA effect is more significant than the predicted $2 p 2 h$ effect. These data appear sensitive to 
details of the CCQE vs. $2 p 2 h$ processes not yet exposed within the available models, details such as those $[18,19,55]$ that go beyond the Fermi gas.

This $2 p 2 h$ tune comes with three other variations that treat the final state nucleon content as uncertain. Instead of enhancing all $2 p 2 h$ events, the first variation enhances only those generated for $p n$ initial state nucleon pairs, which translate to $p p$ final states for the neutrino case in the fit and $n n$ for the antineutrino case where we apply the tuned parameters. The next variation enhances reactions that are not on $p n$ initial state pairs, which lead to $p n$ final states. Finally, the third variation enhances CCQE events at these kinematics. In addition to testing these variations against the reconstructed data, they are used as an uncertainty applied later when producing a double-differential cross section.

This sample also includes a significant component at and beyond the $\Delta$ resonance peak, which remains poorly described by these model variations. The shortcoming of the model for these low $Q^{2}=q_{3}^{2}-q_{0}^{2} \approx 0$ events shows up on the far right of the distributions in Fig. 3. Similar mismodeling of the resonance-region rate has been previously reported in measurements on mineral oil by MiniBooNE [56,57], in MINERvA's pion final state samples $[49,58,59]$, in the neutrino version of this analysis [6], and in a resonance-rich neutrino $+\mathrm{Fe}$ sample from MINOS [53]. The latter used a calorimetric sample as a sideband and tuned an ad hoc, low $Q^{2}$ suppression to the data in order to improve the estimate of the resonance background in their CCQE analysis. At $Q^{2}=0$, the rate is $40 \%$ of nominal and becomes no suppression by $Q^{2}=0.7(\mathrm{GeV} / c)^{2}$. Applying the MINOS parametrization improves the description of these MINERvA data for some of those bins at high $q_{3}$, but the suppression goes too far and produces a model deficit in the highest energy bins of the low $q_{3}$ panel. These bins in Fig. 3 were already well described, and the $\chi^{2}$ reflects that the agreement worsens. Either the single-parameter $Q^{2}$ weight or the tuning to neutrino $+\mathrm{Fe}$ data is not adequate to describe the two dimensional kinematics of these antineutrino + $\mathrm{CH}$ samples.

To allow the development and testing of improved models, this distribution is unfolded to produce a double differential cross section $d^{2} \sigma / d E_{\text {avail }} d q_{3}$, shown in Fig. 4 and tabulated in the Supplemental Material [60]. The procedure is the same as in [50], Secs. VIIB and VIII, and it uses [61-63] but with three iterations. The resolution for $q_{3}$ in Fig. 2 is with an rms near 23\% throughout and slowly changing with $q_{0}$. The reconstructed available energy is the sum of a component from charged hadron and electromagnetic energy deposits with a central peak of $30 \%$ resolution but a rms of $40 \%$ as in the neutrino case [6]. Then, the random tens of MeV energy from about half of the final state neutrons further degrade the resolution to

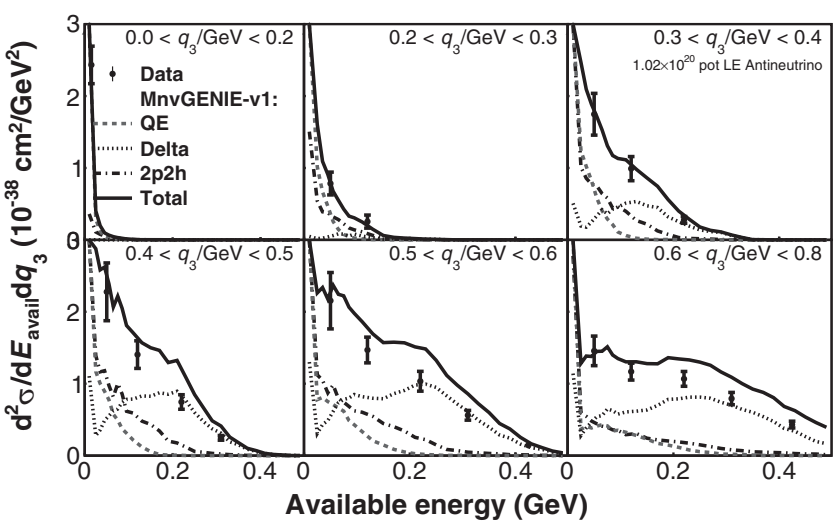

FIG. 4. Unfolded $d^{2} \sigma / d E_{\text {avail }} d q_{3}$ cross section per nucleon compared to the model with RPA and tuned $2 p 2 h$ components. The breakdown of the predicted QE (dashed), $2 p 2 h$ (dot-dashed), and $\Delta$ resonance (dotted) portions are shown. To show the detail, the data and model prediction for the first bin (dominated by neutron-only final states) are not shown; they are far off the top of the plot with values between 9 and $17 \times 10^{-42} \mathrm{~cm}^{-2} / \mathrm{GeV}^{2}$ per nucleon.

Fig. 2. The $25 E_{\text {avail }}, q_{3}$ bins were chosen based on these resolutions.

The largest fractional uncertainties in half the bins, up to $14 \%$, come from variations on the $2 p 2 h$ enhancement used in the unfolding model. When the enhancement is formed only from events with $p n$ initial state pairs (preferentially $n n$ final states in the antineutrino case), the migration matrix has a higher probability to put events in the low $E_{\text {avail }}$ bins. The opposite is true when the enhancement only adds $p p$ initial state pairs. The difference to the nominal cross section is added to the uncertainty. The uncertainty assigned to GENIE's intranuclear rescattering model is also large because it modifies the unfolding model in this steep region of the cross section. These uncertainties are of similar size to the flux uncertainty, suggesting that a future cycle of cross section model improvements could yield an even more precise cross section. The breakdown of uncertainties and the full covariance matrix are presented in the Supplemental Material [60].

In conclusion, the hadronic energy spectrum from a sample of low momentum transfer antineutrino interactions suggests the need for a RPA-like suppression [17] of quasielastic events, relative to a Fermi gas model. In addition, an enhancement on top of the IFIC Valencia $2 p 2 h$ component $[8,45]$ is essential to supply the observed event rate in the region between the CCQE and $\Delta$ peaks. We add to the evidence for a low $Q^{2}$ suppression of resonance events by demonstrating that the MINOS parametrization [53] offers some improvement to the $\chi^{2}$. The model elements above were tested or fit to describe lepton and hadronic components of neutrino data. Critical for oscillation experiments in this neutrino energy range, they offer a similarly good description of these antineutrino data. 
This document was prepared by members of the MINERvA collaboration using the resources of the Fermi National Accelerator Laboratory (Fermilab), a U.S. Department of Energy, Office of Science, HEP User Facility. Fermilab is managed by the Fermi Research Alliance, LLC (FRA), acting under Contract No. DE-AC02-07CH11359. These resources included support for the MINERvA construction project, and support for construction was also granted by the United States National Science Foundation under Award No. PHY0619727 and by the University of Rochester. Support for participating scientists was provided by NSF and DOE (USA) by CAPES and CNPq (Brazil), by CoNaCyT (Mexico), by Proyecto Basal FB 0821, CONICYT PIA ACT1413, Fondecyt 3170845 and 11130133 (Chile), by CONCYTEC, DGI-PUCP, and UDI/IGI-UNI (Peru), and by the Latin American Center for Physics (CLAF). We thank the MINOS Collaboration for the use of its near detector data. Finally, we thank the staff of Fermilab for the support of the beam line, the detector, and computing infrastructure.

*Present address: Iowa State University, Ames, Iowa 50011, USA.

'Present address: Lawrence Berkeley National Laboratory, Berkeley, California 94720, USA.

*Present address: University of Michigan, Ann Arbor, Michigan 48109, USA.

${ }^{\S}$ Present address: University College London, London WC1E 6BT, United Kingdom.

"Present address: Tufts University, Medford, Massachusetts 02155, USA.

[1] P. Adamson et al. (NOvA Collaboration), Phys. Rev. D 93, 051104 (2016).

[2] P. Adamson et al. (NOvA Collaboration), Phys. Rev. Lett. 116, 151806 (2016).

[3] R. Acciarri et al. (Fermilab Short Baseline Collaboration), arXiv: 1503.01520.

[4] C. Adams et al. (LBNE Collaboration), arXiv:1307.7335.

[5] R. Acciarri et al. (DUNE Collaboration), arXiv:1512.06148.

[6] P. A. Rodrigues et al. (MINERvA Collaboration), Phys. Rev. Lett. 116, 071802 (2016).

[7] M. Martini, M. Ericson, G. Chanfray, and J. Marteau, Phys. Rev. C 80, 065501 (2009).

[8] J. Nieves, I. Ruiz Simo, and M. Vicente Vacas, Phys. Rev. C 83, 045501 (2011).

[9] R. Gonzalez-Jimenez, G. D. Megias, M. B. Barbaro, J. A. Caballero, and T. W. Donnelly, Phys. Rev. C 90, 035501 (2014).

[10] G. D. Megias, J. E. Amaro, M. B. Barbaro, J. A. Caballero, T. W. Donnelly, and I. R. Simo, Phys. Rev. D 94, 093004 (2016).

[11] T. Van Cuyck, N. Jachowicz, R. Gonzalez-Jimenez, J. Ryckebusch, and N. Van Dessel, Phys. Rev. C 95, 054611 (2017).
[12] I. Ruiz Simo, J. E. Amaro, M. B. Barbaro, J. A. Caballero, G. D. Megias, and T. W. Donnelly, Ann. Phys. (Amsterdam) 388, 323 (2018).

[13] J. W. Lightbody and J. S. O'Connell, Comput. Phys. 2, 57 (1988).

[14] J. T. Sobczyk, Phys. Rev. C 86, 015504 (2012).

[15] J. Schwehr, D. Cherdack, and R. Gran, arXiv:1601.02038.

[16] K. Gallmeister, U. Mosel, and J. Weil, Phys. Rev. C 94, 035502 (2016).

[17] J. Nieves, J. E. Amaro, and M. Valverde, Phys. Rev. C 70, 055503 (2004).

[18] V. Pandey, N. Jachowicz, T. Van Cuyck, J. Ryckebusch, and M. Martini, Phys. Rev. C 92, 024606 (2015).

[19] J. Nieves and J. E. Sobczyk, Ann. Phys. (Amsterdam) 383, 455 (2017).

[20] R. A. Smith and E. J. Moniz, Nucl. Phys. B43, 605 (1972).

[21] P. Adamson et al., Nucl. Instrum. Methods Phys. Res., Sect. A 806, 279 (2016).

[22] S. Agostinelli et al., Nucl. Instrum. Methods Phys. Res., Sect. A 506, 250 (2003).

[23] J. Allison et al., IEEE Trans. Nucl. Sci. 53, 270 (2006).

[24] L. Aliaga et al. (MINERvA Collaboration), Phys. Rev. D 94, 092005 (2016); 95, 039903 (2017)].

[25] C. Alt et al. (NA49 Collaboration), Eur. Phys. J. C 49, 897 (2007).

[26] S. P. Denisov, S. V. Donskov, Yu. P. Gorin, R. N. Krasnokutsky, A. I. Petrukhin, Yu. D. Prokoshkin, and D. A. Stoyanova, Nucl. Phys. B61, 62 (1973).

[27] A. S. Carroll et al., Phys. Lett. B 80, 319 (1979).

[28] J. V. Allaby et al. (IHEP-CERN Collaboration), Phys. Lett. B 30, 500 (1969).

[29] J. Park et al. (MINERvA Collaboration), Phys. Rev. D 93, 112007 (2016).

[30] D. G. Michael et al. (MINOS Collaboration), Nucl. Instrum. Methods Phys. Res., Sect. A 596, 190 (2008).

[31] L. Aliaga et al. (MINERvA Collaboration), Nucl. Instrum. Methods Phys. Res., Sect. A 743, 130 (2014).

[32] L. Aliaga et al. (MINERvA Collaboration), Nucl. Instrum. Methods Phys. Res., Sect. A 789, 28 (2015).

[33] W. P. Abfalterer, F. B. Bateman, F. S. Dietrich, R. W. Finlay, R. C. Haight, and G. L. Morgan, Phys. Rev. C 63, 044608 (2001).

[34] C. Andreopoulos et al., Nucl. Instrum. Methods Phys. Res., Sect. A 614, 87 (2010).

[35] J. D. Devan, Measurement of neutrino and antineutrino charged-current inclusive cross sections with the MINERvA detector, Ph.D. thesis, College of William and Mary, 2015.

[36] C. H. Llewellyn Smith, Phys. Rep. 3, 261 (1972).

[37] R. Bradford, A. Bodek, H. S. Budd, and J. Arrington, Nucl. Phys. B, Proc. Suppl. 159, 127 (2006).

[38] D. Rein and L. M. Sehgal, Ann. Phys. (N.Y.) 133, 79 (1981).

[39] A. Bodek, I. Park, and U.-K. Yang, Nucl. Phys. B, Proc. Suppl. 139, 113 (2005).

[40] P. Rodrigues, C. Wilkinson, and K. McFarland, Eur. Phys. J. C 76, 474 (2016).

[41] C. Wilkinson, P. Rodrigues, S. Cartwright, L. Thompson, and K. McFarland, Phys. Rev. D 90, 112017 (2014).

[42] A. Higuera et al. (MINERvA Collaboration), Phys. Rev. Lett. 113, 261802 (2014). 
[43] A. Mislivec et al. (MINERvA Collaboration), Phys. Rev. D 97, 032014 (2018).

[44] C. Berger and L. M. Sehgal, Phys. Rev. D 79, 053003 (2009).

[45] R. Gran, J. Nieves, F. Sanchez, and M. J. Vicente Vacas, Phys. Rev. D 88, 113007 (2013).

[46] R. Gran, arXiv:1705.02932.

[47] P. A. Rodrigues et al. (MINERvA Collaboration) (to be published).

[48] M. Betancourt et al. (MINERvA Collaboration), Phys. Rev. Lett. 119, 082001 (2017).

[49] O. Altinok et al. (MINERvA Collaboration), Phys. Rev. D 96, 072003 (2017).

[50] C. E. Patrick et al. (MINERvA Collaboration), Phys. Rev. D 97, 052002 (2018).

[51] X.-G. Lu, M. Betancourt, T. Walton et al., arXiv: 1805.05486.

[52] M. Valverde, J. E. Amaro, and J. Nieves, Phys. Lett. B 638, 325 (2006).

[53] P. Adamson et al. (MINOS Collaboration), Phys. Rev. D 91, 012005 (2015).

[54] A. S. Meyer, M. Betancourt, R. Gran, and R. J. Hill, Phys. Rev. D 93, 113015 (2016).
[55] M. Martini, N. Jachowicz, M. Ericson, V. Pandey, T. Van Cuyck, and N. Van Dessel, Phys. Rev. C 94, 015501 (2016).

[56] A. A. Aguilar-Arevalo et al. (MiniBooNE), Phys. Rev. D 83, 052007 (2011).

[57] A. A. Aguilar-Arevalo et al. (MiniBooNE), Phys. Rev. D 83, 052009 (2011).

[58] C. L. McGivern et al. (MINERvA Collaboration), Phys. Rev. D 94, 052005 (2016).

[59] B. Eberly et al. (MINERvA Collaboration), Phys. Rev. D 92, 092008 (2015).

[60] See Supplemental Material at http://link.aps.org/ supplemental/10.1103/PhysRevLett.120.221805, for tabulated flux, cross sections, covariance matrix for the uncertainty, and a breakdown of the components of the uncertainty.

[61] G. D’Agostini, Nucl. Instrum. Methods Phys. Res., Sect. A 362, 487 (1995).

[62] G. D’Agostini, arXiv:1010.0632.

[63] T. Adye, arXiv:1105.1160.

Correction: The previously published Figure 4 contained an error in the units and has been replaced. 